\title{
A GENERALIZATION OF BERNSTEIN'S THEOREM AND A DIFFERENTIAL INVERSION FORMULA
}

\author{
BY \\ W. J. STUDDEN( $\left.{ }^{1}\right)$
}

1. Introduction. A function $\phi(t)$ defined on $(0, \infty)$ which satisfies $(-1)^{n} \phi^{(n)}(t) \geqq 0$ for all $t>0$ and $n=0,1, \ldots$ is called completely monotonic on $(0, \infty)$. The classical theorem of Bernstein states that $\phi(t)$ is completely monotonic on $(0, \infty)$ if and only if $\phi(t)$ has a representation of the form

$$
\phi(t)=\int_{0}^{\infty} e^{-x t} d \mu(x), \quad t>0
$$

where $\mu(x)$ a Borel measure on [0, $\infty$ ). (See e.g. Widder [6].)

A considerable number of extensions and generalizations of the above result have been given. After a suitable change of variable the integral transform (1.1) can be written as a convolution on $(-\infty, \infty)$ and is a special case of a large class of convolution transforms which can be inverted by means of a sequence of differential operators. For the special case (1.1) this inversion reduces to

$$
\lim _{n \rightarrow \infty} \frac{(-1)^{n}}{n !}\left(\frac{n}{t}\right)^{n+1} \phi^{(n)}\left(\frac{n}{t}\right)=f(t)
$$

when $d \mu(x)=f(x) d x$ and mild conditions are imposed on $f$. The convolution transforms are studied extensively in Hirschman and Widder [2].

Equation (1.1) is also a special case of representation theorems obtained by Choquet [1] (see also Phelps [5] and references therein) for functions which are alternating of order $\infty$. For such classes of functions it is shown that the extremal functions are those for which $\phi(t+s)=\phi(t) \phi(s)$ and that semigroup operations play an important role. If we consider the convex set of completely monotonic functions on $(0, \infty)$ such that $\phi(0+)=1$ then the extreme points of this set are the functions $\phi_{x}(t)=e^{-x t}$ for $x \geqq 0$. Various other extensions and numerous applications are available in the literature.

The purpose of this paper is to consider a class of generalized completely monotonic functions on $(0, \infty)$, defined in terms of certain differential inequalities and to establish a representation theorem and an inversion formula analogous to (1.1) and (1.2).

Received by the editors January 29, 1968 and, in revised form, August 19, 1968.

${ }^{1}{ }^{1}$ This research was partly supported by the Office of Naval Research contract NONR1100(26) at Purdue University. Reproduction in whole or in part is permitted for any purposes of the United States Government. 
The basic definitions and the theorems are stated in $\$ 2$. $\$ 3$ contains some preliminary lemmas which may be of independent interest while the proofs of the main theorems are contained in $\$ 4$.

The author wishes to express his sincere thanks to Professor Samuel Karlin for introducing him to the subject material of the paper. Thanks are also due to Professor James W. Yackel for a number of helpful discussions and comments.

2. Definitions and statement of theorems. Let $\left\{w_{i}(t)\right\}_{i=0}^{\infty}$ be an infinite sequence of functions which are positive on $[0, \infty)$, each of class $C^{\infty}[0, \infty)$, and define a sequence of differential operators

$$
D_{i} f(t)=\frac{d}{d t} \frac{f(t)}{w_{i}(t)}, \quad i=0,1,2, \ldots
$$

Definition 1. A function $\phi(t)$ defined on $(0, \infty)$ is called a "generalized completely monotonic" function on $(0, \infty)$ (abbreviated GCM on $(0, \infty))$ with respect to $\left\{w_{i}(t)\right\}_{i=0}^{\infty}$ provided $\phi(t)$ is of class $C^{\infty}$ on $(0, \infty)$ and satisfies the inequalities

$$
\phi(t) \geqq 0 \text { and }(-1)^{n+1} D_{n} D_{n-1} \cdots D_{0} \phi(t) \geqq 0
$$

for all $t \in(0, \infty), n=0,1,2, \ldots$

In a recent paper Karlin and Ziegler [4] (see also Karlin and Studden [3]) consider the class of generalized absolutely monotonic functions (GAM) on an interval $(a, b)$ defined by requiring that $\phi(t) \geqq 0$ and $D_{n} D_{n-1} \cdots D_{0} \phi(t) \geqq 0$ for all $t \in(a, b)$ and $n=0,1,2, \ldots$ Introducing the special functions

$$
\begin{aligned}
u_{n}(t)=w_{0}(t) \int_{a}^{t} w_{1}\left(\xi_{1}\right) \int_{a}^{\xi_{1}} w_{2}\left(\xi_{2}\right) \cdots \int_{a}^{\xi_{n-1}} w_{n}\left(\xi_{n}\right) d \xi_{n} \cdots d \xi_{1}, \\
n=0,1, \ldots, t \in[a, b],
\end{aligned}
$$

they show, under mild assumptions on the sequence $\left\{w_{i}(t)\right\}$, that each GAM function $\phi$ on $(a, b)$ has a representation

$$
\phi(t)=\sum_{n=0}^{\infty} \rho_{n}(a+) u_{n}(t), \quad t \in(a, b),
$$

where

$$
\rho_{0}(t)=\frac{\phi(t)}{w_{0}(t)}, \quad \rho_{n}(t)=\rho_{n}(t ; \phi)=\frac{D_{n-1} \cdots D_{0} \phi(t)}{w_{n}(t)}, \quad n=1,2, \ldots
$$

The function $u_{n}(t)$ is the unique solution of the $(n+1)$ th order differential equation $D_{n} D_{n-1} \cdots D_{0} u=0$ subject to the boundary conditions $u^{(i)}(a)=0, i=0,1, \ldots, n-1$, and $u^{(n)}(a)=\prod_{i=0}^{n} w_{i}(a)$. The representation (2.3) corresponds to an expansion of $\phi(t)$ about a finite point in terms of $\left\{u_{n}\right\}$ while the representation (1.1) and the analog we shall obtain below in (2.9) correspond to expansions at $\infty$. It should be noted here and in the following that the classical situation corresponds to the special choice $w_{i}(t) \equiv 1, i=0,1, \ldots$ In this case $D_{n-1} \cdots D_{0} \phi(t)=\phi^{(n)}(t)$ and $u_{n}(t)=(t-a)^{n} / n !$. 
We shall have need of an alternate definition of the convex cone of GCM functions which is expressed in terms of certain determinant inequalities.

Definition 2. A function $\phi$ defined on $(0, \infty)$ belongs to $\kappa\left(u_{0}, u_{1}, \ldots, u_{n}\right)$ and is called convex with respect to $u_{0}, u_{1}, \ldots, u_{n}$ if for every set of $n+2$ points $\left\{t_{i}\right\}_{i=1}^{n+2}$ satisfying $0<t_{1}<t_{2}<\cdots<t_{n+2}$ the determinant inequality

prevails.

$$
(-1)^{n+1}\left|\begin{array}{ccc}
u_{0}\left(t_{1}\right) & \cdots & u_{0}\left(t_{n+2}\right) \\
u_{1}\left(t_{1}\right) & \cdots & u_{1}\left(t_{n+2}\right) \\
\vdots & & \\
u_{n}\left(t_{1}\right) & \cdots & u_{n}\left(t_{n+2}\right) \\
\phi\left(t_{1}\right) & \cdots & \phi\left(t_{n+2}\right)
\end{array}\right| \geqq 0
$$

Consider the intersection cone

$$
\mathscr{C}=\kappa^{+} \cap\left[\bigcap_{n=0}^{\infty} \kappa\left(u_{0}, \ldots, u_{n}\right)\right]
$$

where $\kappa^{+}$denotes the cone of continuous nonnegative functions on $(0, \infty)$. It is proved in Karlin and Studden [3] (see also Karlin and Ziegler [4]) that the convex cone $\mathscr{C}$ coincides with the class of GCM functions. Note that when $w_{i}(t) \equiv 1$ the cones $\kappa(1)$ and $\kappa(1, t)$ correspond to the nonincreasing and convex functions respectively.

Throughout the entire paper we shall assume that the sequence $\left\{w_{i}(t)\right\}_{i=0}^{\infty}$ is such that there exists a function $f(t)$ defined on $[0, \infty)$ such that

1. $0<f(t)<1, t \in[0, \infty)$,

2. $1-f(t) \leqq w_{i}(t) \leqq 1+f(t), t \in[0, \infty), i=1,2, \ldots$,

3. $\lim _{t \rightarrow \infty} f(t)=0$,

4. $\int_{0}^{\infty} f(t) d t<\infty$.

We assume further that $w_{0}(t) \equiv 1$ otherwise we would replace $\phi$ by $\phi / w_{0}$. These assumptions state that the functions $w_{i}(t)$ are asymptotically one at $t=\infty$ in a rather strong sense.

Let

$$
\begin{aligned}
\Psi_{n}(t ; x) & =\int_{t}^{x} w_{n}\left(\xi_{n}\right) \int_{t}^{\xi_{n}} w_{n-1}\left(\xi_{n-1}\right) \cdots \int_{t}^{\xi_{2}} w_{1}\left(\xi_{1}\right) d \xi_{1} \cdots d \xi_{n}, \quad 0 \leqq t \leqq x, \\
& =0, \quad t>x
\end{aligned}
$$

and $\Psi_{0}(t ; x)=1$ for $t \leqq x$ and 0 for $t>x$. The analog of $e^{-x t}$ for the GCM functions is

$$
\Psi(t ; x)=e^{-x t} P(t ; x) / P(0 ; x), \quad 0 \leqq t<\infty, \quad 0 \leqq x<\infty
$$

where

$$
P(t ; x)=1+\int_{t}^{\infty} e^{-x(\xi-t)} \sum_{k=1}^{\infty} x^{k} f_{k}(\xi) \Psi_{k-1}(t ; \xi) d \xi
$$

and $f_{k}(\xi)=w_{k}(\xi)-1, k=1,2, \ldots$ 
THEOREM 1. Under the assumptions $1-4$, a function $\phi(t)$ defined on $(0, \infty)$ is a GCM function if and only if $\phi(t)$ possesses a representation of the form

$$
\phi(t)=\int_{0}^{\infty} \Psi(t ; x) d \mu(x), \quad t \in(0, \infty),
$$

where $\mu$ is a Borel measure on $[0, \infty)$.

We note that in the situation where $w_{k}(t)=1+g(t), k=1,2, \ldots$, for some fixed function $g$ the expression (2.7) reduces to

$$
\Psi(t ; x)=\exp \{-x(t+G(t))\}
$$

where $G(t)=\int_{0}^{t} g(\xi) d \xi$. The expression (2.9) in this case may be derived directly from (1.1).

In order to state the analog of (1.2) we introduce the operators $L_{n}$ defined, at least on GCM functions, by

$$
\left(L_{n} \phi\right)(t)=(-1)^{n+1} P(0, t) \frac{n}{t^{2}}\left(\frac{n}{t}\right)^{n} \frac{1}{n !} D_{n} \cdots D_{0} \phi\left(\frac{n}{t}\right), \quad t>0 .
$$

THEOREM 2. Suppose that for a given measure $\mu$ on $[0, \infty)$ the integral

$$
\phi(t)=\int_{0}^{\infty} \Psi(t ; x) d \mu(x)
$$

converges for all $t \geqq a_{0} \geqq 0$ and that $a$ and $b$ are continuity points of $\mu$. Then under assumptions $1-4$

$$
\lim _{n \rightarrow \infty} \int_{a}^{b}\left(L_{n} \phi\right)(t) d t=\mu\{(a, b)\} .
$$

Theorem 2 immediately implies that the measure $\mu$ corresponding to each GCM function $\phi(t)$ is unique. We shall prove the representation in Theorem 1 first for the case where $\phi(0+)<\infty$ and then extend the result using Theorem 2. The more precise analog to (1.2) is given below under conditions which are clearly stronger than necessary.

THEOREM 3. If

$$
\phi(t)=\int_{0}^{\infty} \Psi(t ; x) f(x) d x .
$$

where $f(x)$ is bounded and continuous on $[0, \infty)$ then

$$
\lim _{n \rightarrow \infty} L_{n} \phi(t)=f(t), \quad t>0 .
$$

With the aid of Theorems 1 and 2 the following result is immediate.

THEOREM 4. Let $K$ denote the convex set of GCM functions for which $\phi(0+)=1$. Then the extreme points of $K$ are the functions $\phi_{x}(t)=\Psi(t ; x), x \geqq 0$. (The extremal rays of the cone of GCM functions are rays $\left.\left\{\lambda \phi_{x}(t) \mid \lambda \geqq 0\right\}.\right)$

Note that the set $K$ consists of those GCM functions for which $\int_{0}^{\infty} d \mu=1$ and the representation (2.9) is an extreme point representation. 


\section{Preliminary lemmas.}

LEMMA 1. Under assumptions 1-4

$$
(\xi-t-F(\xi)+F(t))^{k} / k ! \leqq \Psi_{k}(t ; \xi) \leqq(\xi-t+F(\xi)-F(t))^{k} / k !
$$

and

$$
\exp \{-x[F(\infty)-F(t)]\} \leqq P(t ; x) \leqq \exp \{x[F(\infty)-F(t)]\}
$$

Proof. The bounds in equation (3.1) follow from assumption 2. To verify (3.2) we apply (3.1) to obtain

$$
\begin{aligned}
P(t ; x) & \leqq 1+\int_{t}^{\infty} e^{-x(\xi-t)} x f(\xi) \exp \{x(\xi-t)+x[F(\xi)-F(t)]\} d \xi \\
& =\exp \{x[F(\infty)-F(t)]\} .
\end{aligned}
$$

The left side of (3.2) follows similarly.

LEMMA 2. Under assumptions 1-4

$$
D_{n} \cdots D_{0} \Psi(t ; x)=(-x)^{n+1} w_{n+1}(t) e^{-x t} P_{n}(t ; x) / P(0 ; x), \quad t, x \in[0, \infty),
$$

where the above derivatives are taken with respect to the $t$ variable and

$$
P_{n}(t ; x)=1+\int_{t}^{\infty} e^{-x(\xi-t)} \sum_{k=1}^{\infty} x^{k} f_{n+1+k}(\xi) \Psi_{k-1}(t ; \xi ; n) d \xi
$$

and

$$
\begin{aligned}
& \Psi_{k-1}(t ; \xi ; n) \\
& \quad=\int_{t}^{\xi} w_{n+k}\left(\xi_{k-1}\right) \int_{t}^{\xi_{k-1}} w_{n+k-1}\left(\xi_{k-2}\right) \cdots \int_{t}^{\xi_{2}} w_{n+2}\left(\xi_{1}\right) d \xi_{1} \cdots d \xi_{k-1} .
\end{aligned}
$$

Proof. Since the differentiation is on the $t$ variable the factor $P(0 ; x)$ plays no role. The validity of (3.3) is proven by induction. Since we have assumed $w_{0}(t) \equiv 1$

$$
D_{0}\left(e^{-x t} P(t ; x)\right)=\frac{d}{d t}\left[e^{-x t}+x \int_{x}^{\infty} e^{-x \xi} f_{1}(\xi) d \xi+\int_{t}^{\infty} e^{-x \xi} \sum_{k=2}^{\infty} x^{k} f_{k}(\xi) \Psi_{k-1}(t ; \xi) d \xi\right]
$$

Note that $d \Psi_{k}(t, \xi) / d t=-w_{1}(t) \Psi_{k-1}(t ; \xi ; 1)$ and that differentiation past the integral and sum are readily justified on the basis of Lemma 1 . Therefore

$$
\begin{aligned}
D_{0}\left(e^{-x t} P(t ; x)\right) & =-x e^{-x t}-x e^{-x t} f_{1}(t)-w_{1}(t) \int_{t}^{\infty} e^{-x \xi} \sum_{k=2}^{\infty} x^{k} f_{k}(\xi) \Psi_{k-2}(t+\xi ; 1) d \xi \\
& =-x w_{1}(t)\left[e^{-x t}+\int_{t}^{\infty} e^{-x \xi} \sum_{k=1}^{\infty} x^{k} f_{k+1}(\xi) \Psi_{k-1}(t ; \xi ; 1) d \xi\right] \\
& =-x w_{1}(t) e^{-x t} P_{1}(t ; x) .
\end{aligned}
$$

The induction step is clear from the above considerations. 
REMARK. On the basis of (3.3) it can be established that the functions $\Psi(t ; x)$ have an expansion as in (2.3) where we take $a=0$. That is,

$$
\Psi(t ; x)=1+\sum_{n=1}^{\infty}(-1)^{n} x^{n} \frac{P_{n-1}(0 ; x)}{P(0 ; x)} u_{n}(t)
$$

LEMMA 3. For $x>t \geqq 0$

$$
\Psi_{n}(t ; x)=\frac{(x-t)^{n}}{n !}+\sum_{k=1}^{n} \int_{t}^{x} f_{k}(\xi) \Psi_{k-1}(t ; \xi) \frac{(x-\xi)^{n-k}}{(n-k) !} d \xi
$$

and for $n / x>t \geqq 0$

$$
\begin{array}{r}
\Psi_{n}\left(t ; \frac{n}{x}\right)=\left(\frac{n}{x}-t\right)^{n} \frac{1}{n !}\left\{1+\sum_{k=1}^{n} x^{k} \int_{t}^{n / x} f_{k}(\xi) \Psi_{k-1}(t ; \xi) \frac{n !}{(n-x t)^{k}(n-k) !}\right. \\
\left.\cdot\left[1-\frac{x(\xi-t)}{n-x t}\right]^{n-k} d \xi\right\}
\end{array}
$$

Proof. Equation (3.7) follows readily from (3.6) while (3.7) is proven by induction. The case $n=1$ is immediate. Assuming (3.7) true for $n$ we then have

$$
\begin{aligned}
\Psi_{n+1}(t ; x)= & \int_{t}^{x} w_{n+1}(\eta) \Psi_{n}(t ; \eta) d \eta \\
= & \int_{t}^{x} \Psi_{n}(t ; \eta) d \eta+\int_{t}^{x} f_{n+1}(\eta) \Psi_{n}(t ; \eta) d \eta \\
= & \int_{t}^{x}\left[\frac{(\eta-t)^{n}}{n !}+\sum_{k=1}^{n} \int_{t}^{\eta} f_{k}(\xi) \Psi_{k-1}(t ; \xi) \frac{(\eta-\xi)^{n-k}}{(n-k) !} d \xi\right] d \eta \\
& +\int_{t}^{x} f_{n+1}(\eta) \Psi_{n}(t ; \eta) d \eta .
\end{aligned}
$$

The term $(\eta-t)^{n}$ can be integrated directly. For the $n$ terms in the sum we interchange the order of integration on $\xi$ and $\eta$ and integrate the term $(\eta-\xi)^{n-k}$. We thus have

$$
\Psi_{n+1}(t ; x)=\frac{(x-t)^{n+1}}{(n+1) !}+\sum_{k=1}^{n+1} \int_{t}^{x} f_{k}(\xi) \Psi_{k-1}(t ; \xi) \frac{(x-\xi)^{n+1-k}}{(n+1-k) !} d \xi
$$

which completes the induction.

LEMMA 4. Under the assumptions 1-4 the functions

$$
\begin{aligned}
\Phi_{n}(t ; x) & =\Psi_{n}(t ; n / x) / \Psi_{n}(0, n / x), & & n \geqq t x, \\
& =0, & & n<t x,
\end{aligned}
$$

converge uniformly in $x$ to $\Psi(t ; x)$ for fixed $t \geqq 0$.

Proof. Applying Lemma 9.2, p. 437 of Karlin and Studden [3] with $k=2$ we find that the functions $\Phi_{n}(t ; x)$ are nonincreasing in $x$. If $\lim _{n \rightarrow \infty} \Phi_{n}(t ; x)=\Psi(t ; x)$ 
the uniformity will follow since it is easily seen that the limit function $\Psi(t ; x)$ is continuous in $x$.

Considering equation (3.7) we note that the ratio of the terms $(n / x-t)^{n} / n$ ! and $(n / x)^{n} / n$ ! converges to $e^{-x t}$. It thus suffices to show that

$$
\int_{t}^{n / x} \sum_{k=1}^{n} x^{k} f_{k}(\xi) \Psi_{k-1}(t ; \xi) \frac{n !}{(n-x t)^{k}(n-k) !}\left[1-\frac{x(\xi-t)}{n-x t}\right]^{n-k} d \xi
$$

converges for $t \geqq 0$ to

$$
\int_{t}^{\infty} e^{-x(\xi-t)} \sum_{k=1}^{\infty} x^{k} f_{k}(\xi) \Psi_{k-1}(t ; \xi) d \xi
$$

The integrand $f_{n}(\xi)$ in equation (3.9) has the bound

$$
f_{n}(\xi) \leqq \frac{n x}{n-x t}\left(1+\frac{x(F(\infty)-F(t))}{n-x t}\right)^{n-1} f(\xi) \leqq C f(\xi)
$$

and $\int_{0}^{\infty} f(\xi) d \xi<\infty$. By the Lebesgue dominated convergence theorem it suffices to show that the integrands converge for fixed $\xi, x$ and $t$. We now write the integrand as $\sum_{k=1}^{\infty} a_{n}(k)$ where

$$
\begin{aligned}
a_{n}(k) & =x^{k} f_{k}(\xi) \Psi_{k-1}(t ; \xi) \frac{n !}{(n-x t)^{k}(n-k) !}\left[1-\frac{x(\xi-t)}{n-x t}\right]^{n-k}, \quad n \geqq k \\
& =0, \quad n<k .
\end{aligned}
$$

The term $n ! /(n-x t)^{k}(n-k)$ ! is uniformly bounded in $k$ so that

$$
\left|a_{n}(k)\right| \leqq C x^{k} f(\xi)[(\xi-t)+F(\xi)-f(t)]^{k-1} /(k-1) !
$$

which is summable. Appealing again to the Lebesgue dominated convergence theorem the result follows since

$$
\lim _{n \rightarrow \infty} \frac{n !}{(n-x t)^{k}(n-k) !}=1
$$

and

$$
\lim _{n \rightarrow \infty}\left[1-\frac{x(\xi-t)}{n-x t}\right]^{n-k}=e^{-x(\xi-t)}
$$

4. Proofs of theorems.

Proof of Theorem 2. If the measure $\mu$ is such that $\int_{0}^{\infty} \Psi(t ; x) d \mu(x)<\infty$ for all $t>0$, then on the basis of the inequality (3.2) we have

$$
\infty>\int_{0}^{\infty} \Psi(t ; x) d \mu(x) \geqq \int_{0}^{\infty} \exp \{-x[t+2 F(\infty)]\} d \mu(x)
$$

so that

$$
\int_{0}^{\infty} e^{-A x} d \mu(x)<\infty, \quad A>2 F(\infty)
$$


In this case

$$
\int_{0}^{\infty} x^{n+1} e^{-A x} d \mu(x)<\infty \text { for all } n \text { and } A>2 F(\infty) .
$$

Now by Lemma 2

$$
\begin{aligned}
(-1)^{n+1} D_{n} \cdots D_{0} \Psi(t ; x) & =x^{n+1} w_{n+1}(t) e^{-x t} P_{n}(t ; x) / P(0, x) \\
& \leqq w_{n+1}(t) x^{n+1} \exp \{-x[t-2 F(\infty)]\}
\end{aligned}
$$

Therefore

$$
D_{n} \cdots D_{0} \phi(t)=\int_{0}^{\infty} D_{n} \cdots D_{0} \Psi(t ; x) d \mu(x)
$$

at least for $t>4 F(\infty)$. We then have that

$$
L_{n} \phi(t)=\int_{0}^{\infty} L_{n} \Psi(t ; x) d \mu(x) \text { for } n / t>4 F(\infty)
$$

where

$$
L_{n} \Psi(t ; x)=w_{n+1}\left(\frac{n}{t}\right) P_{n}\left(\frac{n}{t} ; x\right) \frac{P(0 ; t)}{P(0 ; x)} \frac{(n x)^{n+1}}{t^{n+2} n !} e^{-n x / t}
$$

To complete the proof of Theorem 2 it suffices to show that if $g_{n}(x)=\int_{a}^{b} L_{n} \Psi(t ; x) d t$ then

$$
g_{n}(x) \leqq g(x) \text { where } \int_{0}^{\infty} g(x) d \mu(x)<\infty
$$

and

$$
\begin{aligned}
\lim _{n \rightarrow \infty} g_{n}(x) & =1, & & x \in(a, b), \\
& =0, & & x \notin(a, b) .
\end{aligned}
$$

Using the bounds from Lemma 1 we observe that for $t \in[a, b]$

$$
\begin{aligned}
(1-f(n / b)) \exp \{-x[F(\infty)-F(n / b)]\} & \leqq w_{n+1}(n / t) P_{n}(n / t ; x) \\
& \leqq(1+f(n / b)) \exp \{-x[F(\infty)-F(n / b)]\} .
\end{aligned}
$$

Moreover the function

$$
h_{n}(t ; x)=\frac{(n x)^{n+1} e^{-n x / t}}{t^{n+2} n !}
$$

is a density function on $(0, \infty)$ with mean $x$ and variance $x^{2} /(n-1)$, i.e.

$$
\int_{0}^{\infty} h_{n}(t ; x) d t=1, \quad \int_{0}^{\infty} t h_{n}(t ; x) d t=x
$$

and

$$
\int_{0}^{\infty}(t-x)^{2} h_{n}(t ; x) d t=\frac{x^{2}}{n-1}
$$


Since $P(0 ; t)$ is bounded and continuous on $[a, b]$ equation (4.7) follows.

To verify (4.6) we again use Lemma 1 and the expression for $L_{n} \Psi(t ; x)$ in (4.5) to obtain

$$
g_{n}(x) \leqq\left(1+f\left(\frac{n}{b}\right)\right) \exp \{b F(\infty)\} \exp \{x[3 F(\infty)]\} \int_{a}^{b} h_{n}(t ; x) d t
$$

Since $\int_{0}^{\infty} e^{-A x} d \mu(x)<\infty$ for $A>2 F(\infty)$ it suffices to show that

$$
\int_{a}^{b} h_{n}(t ; x) d t \leqq K_{1} \exp \left\{-K_{2} x\right\} \quad \text { where } K_{2}>5 F(\infty)
$$

We note that $\int_{a}^{b} h_{n}(t ; x) d t=1$ for all $x$ so that it suffices to obtain (4.9) for $x$ greater than some number which is independent of $n$. Now

$$
\begin{aligned}
\int_{a}^{b} h_{n}(t ; x) d x & =\int_{a}^{b} \frac{(n x)^{n+1} e^{-n x / t}}{t^{n+2} n !} d t=\int_{n x / b}^{n x / a} \frac{z^{n} e^{-z}}{n !} d z \\
& \leqq e^{-n x / 2 b} \int_{n x / b}^{n x / a} \frac{z^{n} e^{-z / 2}}{n !} d z \leqq e^{-n x / 2 b} \int_{0}^{\infty} \frac{z^{n} e^{-z / 2}}{n !} d z \\
& =e^{-n x / 2 b} 2^{n+1}=2 \exp \{-n x / 2 b+n \log 2\}
\end{aligned}
$$

The exponent is decreasing in $n$ whenever $x>2 b \log 2$. Therefore

$$
\int_{a}^{b} h_{n}(t ; x) d x \leqq 2 \exp \left\{n_{0} \log 2\right\} \exp \left\{-n_{0} x / 2 b\right\}
$$

provided $n \geqq n_{0}$ and $x>2 b \log 2$. Choosing $n_{0}$ such that $n_{0}>2 b(5 F(\infty))$ completes the proof.

Proof of Theorem 3. The proof here proceeds similar to Theorem 2. On the basis of (4.3) we have

$$
L_{n} \phi(t)=\int_{0}^{\infty} L_{n} \Psi(t ; x) f(x) d x \text { for } n / t>4 F(\infty)
$$

where

$$
L_{n} \Psi(t ; x)=w_{n+1}\left(\frac{n}{t}\right) P_{n}\left(\frac{n}{t} ; x\right) \frac{P(0 ; t)}{P(0 ; x)} \frac{n+1}{n} g_{n}(x ; t)
$$

and $g_{n}(x ; t)=n h_{n}(t ; x) /(n+1)$. The function $g_{n}(x ; t) \geqq 0$ satisfies

$$
\begin{gathered}
\int_{0}^{\infty} g_{n}(x ; t) d x=1, \quad \int_{0}^{\infty} x g_{n}(x ; t) d x=\left(\frac{n+2}{n}\right) t=t_{n}, \\
\sigma_{n}(t)=\int_{0}^{\infty}\left(x-t_{n}\right)^{2} g_{n}(x, t) d x=t^{2} \frac{(n+2)}{n^{2}} .
\end{gathered}
$$

Proceeding essentially as in Theorem 2 we write

$$
\begin{aligned}
L_{n} \phi(t) & =\int_{0}^{\infty} L_{n} \Psi(t ; x) f(x) d x \\
& =\int_{\left|x-t_{n}\right|<\varepsilon} L_{n} \Psi(t ; x) f(x) d x+\int_{\left|x-t_{n}\right| \geqq \varepsilon} L_{n} \Psi(t ; x) f(x) d x
\end{aligned}
$$


Considering the integral over $\left|x-t_{n}\right|<\varepsilon$ we observe in (4.10) that the term $w_{n+1}(n / t) P_{n}(n / t ; x)(n+1) / n$ can be made uniformly close to one. Since $[P(0 ; x)]^{-1} f(x)$ is bounded and continuous on $\left|x-t_{n}\right| \leqq \varepsilon$ it follows that the first integral approaches $f(t)$. To verify that the second integral converges to zero we note that

$$
L_{n} \Psi(t ; x) f(x) \leqq(n+1) w_{n+1}(n / t) P(0 ; t) B e^{A x} g_{n}(x, t) / n
$$

where $\sup _{0 \leqq x \leqq \infty} f(x) \leqq B$ and $A \geqq[2 F(\infty)-F(n / t)]$. An argument similar to the above readily shows that

$$
\lim _{n \rightarrow \infty} \int_{\left|x-t_{n}\right| \geqq \varepsilon} e^{A x} g_{n}(x, t) d x=0 .
$$

Proof of Theorem 1. We shall first establish the representation in Theorem 1 for those GCM functions such that $\phi(0+)<\infty$. Once this has been established we can extend the result by noting first that $\phi(t)=\phi(t+\varepsilon)$ for $\varepsilon>0$ has $\phi(0)<\infty$ and is contained in the cone generated by the system $\left\{w_{i}(t+\varepsilon)\right\}_{i=0}^{\infty}$. In this case

$$
\phi(t+\varepsilon)=\int_{0}^{\infty} e^{-x t} \frac{P_{\varepsilon}(t ; x)}{P_{\varepsilon}(0 ; x)} d \mu_{\varepsilon}(x)
$$

where $P_{\varepsilon}(t ; x)$ is defined as in (2.8) using the system $\left\{w_{i}(t+\varepsilon)\right\}$. A change of variable shows that

$$
P_{\varepsilon}(t ; x)=1+\int_{t+\varepsilon}^{\infty} e^{-\varkappa(\xi-\varepsilon-t)} \sum_{k=1}^{\infty} x^{x} f_{k}(\xi) \Psi_{k-1}(t+\varepsilon ; \xi) d \xi
$$

It then follows from (4.11) that

where

$$
\phi(t)=\int_{0}^{\infty} \Psi(t ; x) d v_{\varepsilon}(x) \text { for } t \geqq \varepsilon
$$

$$
d v_{\varepsilon}(x)=e^{x \varepsilon} P(0 ; x) / P(\varepsilon ; x) d \mu_{\varepsilon}(x) .
$$

However by Theorem 2 the measure corresponding to any $\phi$ is unique so that $d v_{\varepsilon}=d \mu$ is independent of $\varepsilon$ and hence

$$
\phi(t)=\int_{0}^{\infty} \Psi(t ; x) d \mu(x)
$$

We now consider the case where $\phi$ is a GCM function such that $\phi(0+)<\infty$. We shall assume without loss of generality that $\phi(0+)=1$. The case $\phi(0+)=0$ is trivial since $\phi$ is nonincreasing and hence identically zero.

Appealing to [3, p. 387] or by repeated integration by parts we find that for $0<t<c$

$$
\phi(t)-\phi(c)=\int_{t}^{c} \Psi_{n}(t ; x) G_{n+1} \phi(x) d x+\sum_{k=1}^{n} \Psi_{k}(t ; c) \frac{G_{k} \phi(c)}{w_{k}(c)}
$$

where

$$
G_{k} \phi(c)=(-1)^{k} D_{k-1} \cdots D_{0} \phi(c), \quad k=1,2, \ldots
$$


We first show that the $k$ terms in the sum on the right side vanish as $c \rightarrow \infty$. Since $G_{k} \phi(x) / w_{k}(x)$ is nonnegative and nonincreasing on $(0, \infty)$ we have

$$
\begin{aligned}
\frac{G_{k} \phi(c)}{w_{k}(c)} \leqq & \frac{2}{c} \int_{c / 2}^{c} \frac{G_{k} \phi(x)}{w_{k}(x)} d x \\
& \leqq \frac{2}{c m} \int_{c / 2}^{c} G_{k} \phi(x) d x \quad\left(0<m=\min _{0 \leqq x<\infty}[1-f(t)]\right) \\
= & \frac{2}{c m}\left[\frac{G_{k-1} \phi(c / 2)}{w_{k-1}(c / 2)}-\frac{G_{k-1} \phi(c)}{w_{k-1}(c)}\right] \\
& \leqq \frac{2}{c m} \frac{G_{k-1} \phi(c / 2)}{w_{k-1}(c / 2)} \\
& \vdots \\
& \leqq \frac{A_{k}}{c^{k}}\left[\phi\left(\frac{c}{2^{k}}\right)-\phi\left(\frac{c}{2^{k-1}}\right)\right]
\end{aligned}
$$

where $A_{k}$ is independent of $c$. The above inequality together with the fact that

$$
\Psi_{k}(t ; c) \leqq(c+F(\infty))^{k} / k !
$$

implies that

$$
\lim _{c \rightarrow \infty} \frac{G_{k} \phi(c)}{w_{k}(c)} \Psi_{k}(t ; c)=0, \quad k=1,2, \ldots,
$$

since $\lim _{c \rightarrow \infty} \phi\left(c / 2^{k}\right)=\phi(\infty)$ exists.

The representation (4.12) thus reduces to

$$
\begin{aligned}
\phi(t)-\phi(\infty) & =\int_{t}^{\infty} \Psi_{n}(t ; x) G_{n+1} \phi(x) d x \\
& =\int_{t}^{\infty} \frac{\Psi_{n}(t ; x)}{\Psi_{n}(0 ; x)} \Psi_{n}(0 ; x) G_{n+1} \phi(x) d x \\
& =\int_{0+}^{n / t} \frac{\Psi_{n}(t ; n / x)}{\Psi_{n}(0 ; n / x)} d \mu_{n}(x)
\end{aligned}
$$

where

$$
d \mu_{n}(x)=\left(n / x^{2}\right) \Psi_{n}(0 ; n / x) G_{n+1} \phi(x) d x .
$$

The measures $\mu_{n}(x)$ are uniformly bounded since $\int_{0+}^{\infty} d \mu_{n}(x) \leqq \phi(0+)-\phi(\infty)$. By the Helly theorem we select a subsequence $\mu_{n_{i}}$ converging to $\mu^{*}$. By Lemma 4 the functions

$$
\begin{aligned}
\Phi_{n}(t ; x) & =\Psi_{n}(t ; n / x) / \Psi_{n}(0 ; n / x), & & n \geqq t x, \\
& =0, & & n<t x,
\end{aligned}
$$

converge uniformly to $\Psi(t ; x)$ for fixed $t>0$. Therefore

$$
\phi(t)-\phi(\infty)=\int_{0+}^{\infty} \Psi(t ; x) d \mu^{*}(x) .
$$


If we define $\mu$ by adding mass $\phi(\infty)$ to $\mu^{*}$ at $x=0$ we obtain the representation

$$
\phi(t)=\int_{0}^{\infty} \Psi(t ; x) d \mu(x)
$$

Thus every GCM function possesses a representation of the form (2.9). To verify the reverse implication we appeal to the alternate Definition 2 of the GCM functions. On the basis of Theorem 4.2 on p. 400 of [3] the function $\phi_{n}(t)$ $=\Psi_{n}(t ; n / x) / \Psi_{n}(0 ; n / x)$ is contained in the cone $\kappa^{+} \cap\left[\bigcap_{k=0}^{n} \kappa\left(u_{0}, \ldots, u_{k}\right)\right]$ so the $\Psi(t ; x)$ is a GCM function. If the integral (2.9) converges for $t>0$ then the inequalities (2.4) are satisfied and $\phi$ is a GCM function.

\section{REFERENCES}

1. Gustave Choquet, Theory of capacities, Ann. Inst. Fourier Grenoble 5 (1955), 131-292.

2. I. I. Hirschman and D. V. Widder, The convolution transform, Princeton Univ. Press, Princeton, N. J., 1955.

3. S. Karlin and W. J. Studden, Tchebycheff systems: with applications in analysis and statistics, Interscience, New York, 1966.

4. S. Karlin and Zvi Ziegler, Generalized absolutely monotone functions, Israel J. Math. 3 (1965), 173-180.

5. R. R. Phelps, Lectures on Choquet's theorem, Van Nostrand, Princeton, N. J., 1966.

6. D. V. Widder, The Laplace transform, Princeton Univ. Press, Princeton, N. J., 1946.

PURdue University,

LAFAYETTE, INDIANA 